Agnès Desmazières, L'inconscient au paradis. Comment les catholiques ont reçu la psychanalyse (1920-1965)

Paris, Payot, 2011, 270 p.

Françoise Champion

URL : http://journals.openedition.org/assr/24517

DOI : $10.4000 /$ assr. 24517

ISSN : $1777-5825$

Éditeur

Éditions de l'EHESS

Édition imprimée

Date de publication : 30 décembre 2012

Pagination : 158

ISSN : 0335-5985

Référence électronique

Françoise Champion, «Agnès Desmazières, L'inconscient au paradis. Comment les catholiques ont reçu la psychanalyse (1920-1965) », Archives de sciences sociales des religions [En ligne], 160 | octobredécembre 2012, mis en ligne le 28 février 2013, consulté le 21 septembre 2020. URL : http:// journals.openedition.org/assr/24517; DOI : https://doi.org/10.4000/assr.24517

Ce document a été généré automatiquement le 21 septembre 2020.

(c) Archives de sciences sociales des religions 


\section{Agnès Desmazières, L'inconscient au paradis. Comment les catholiques ont reçu la psychanalyse (1920-1965)}

Paris, Payot, 2011, 270 p.

Françoise Champion

\section{RÉFÉRENCE}

Agnès Desmazières, L'inconscient au paradis. Comment les catholiques ont reçu la psychanalyse (1920-1965), Paris, Payot, 2011, 270 p. 
1 Le titre de l'ouvrage, plaisant et quelque peu énigmatique, est précisé par son soustitre, Comment les catholiques ont reçu la psychanalyse. En parlant de catholiques au pluriel, Agnès Desmazières souligne qu'il ne peut être question de parler « du catholicisme» tant sont diverses les conceptions et prises de position des catholiques à l'égard de la psychanalyse, et ce, même lorsqu'il y a des condamnations de la hiérarchie catholique allant jusqu'à des mises à l'index d'ouvrages. À suivre l'auteur ellemême, elle aurait pu aussi parler des psychanalyses : sont en effet également très diverses les psychanalyses que l'on voit se déployer au fil de l'ouvrage, y compris lorsqu'on s'en tient à la pensée de Freud, car l'écart est grand entre sa première et sa seconde topique. Pour continuer à aborder l'ouvrage tout

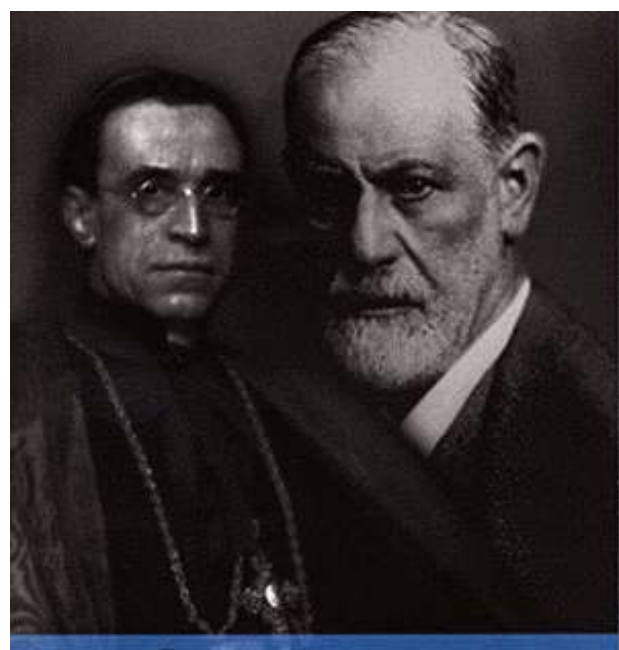

Agnès Desmazières

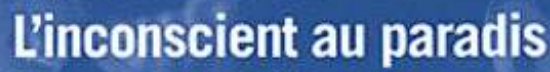

Comment les catholiques ont reçu la psychanalyse

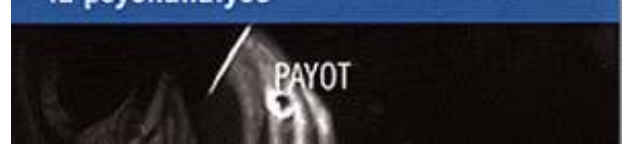
d'abord à travers son titre, indiquons encore qu'il ne s'agit pas seulement d'une réception de «la » psychanalyse par les catholiques, mais que ceux-ci ont pu être des acteurs actifs dans la construction de certaines formes de psychanalyse.

2 L'enquête historique se fonde sur des livres et des articles tant de psychanalystes que de théologiens, ainsi que sur les archives secrètes du Vatican.

3 Les enjeux des nouveaux savoirs et nouvelles pratiques psychanalytiques auxquels les catholiques sont confrontés, avec lesquels ils dialoguent et qu'ils contribuent donc aussi à construire, concernent, pour le dire globalement, la libéralisation des mœurs et, un peu plus précisément - en ignorant les entremêlements et recouvrements des thématiques listées -, la sexualité, la jouissance, la masturbation, l'abstinence des prêtres, la pédagogie, la volonté, le péché, la culpabilité, la conscience morale, la responsabilité morale, la sublimation, la médecine, la science, la modernité... Ces enjeux se retrouvent tout au long des cinquante années étudiées dans cet ouvrage, mais chacun se révèle plus ou moins décisif selon les moments : l'auteur entrecroise une approche chronologique - qui structure l'ouvrage - et une approche thématique. On peut directement observer cet entrecroisement dans les titres de chapitres: "La psychanalyse soupçonnée ", "Première acculturation: doctrine et méthode selon Roland Dalbiez », « Partage d'influence. Un nouveau défi : la morale catholique face à la culpabilité freudienne", "La psychanalyse française en ligne de mire ", "La psychologie des profondeurs impose sa présence au Vatican ", "Allégeance psychanalytique ou obédience romaine?» «Psychanalyse et sacerdoce: le nœud gordien ». Chacun des chapitres est amené à faire de longs retours dans le passé, d'autant plus que doivent être présentés les personnages qui entrent alors en scène. Parmi ceux-ci on citera par ordre d'entrée en scène (ou presque): Sigmund Freud, Jacques Maritain, Angelo Hesnard, Édouard Pichon, Oscar Pfister, Carl Jung, Alfred 
Adler, Roland Dalbiez, Bruno de Jésus Marie, Pierre Janet, Heinz Hartmann, Leo Bartemeir, Gregory Zilboorg, Karen Horney, Noel Mailloux, André Godin, Charles-Henri Nodet, Louis Beinaert, Marc Oraison, René Laforgue, Maryse Choisy, Rudoolf Allers, Pierre Teilhard de Chardin, Charles Odier, Margaret Mead, Françoise Dolto, Emmanuel Mounier, Jacques Lacan, Henry Ey, Albert Plé. On rencontre aussi, bien sûr, les papes, Pie XI et Pie XII ; le personnage le plus important de toute l'histoire que nous narre l'auteur est sans doute le très influent président de l'Académie pontificale des sciences, Agostino Gemelli. À travers tous ces noms, on aura remarqué que c'est à une histoire se déroulant entre Amérique (principalement États-Unis et Québec) et Europe (principalement Italie, France, Belgique, Pays-Bas) qu'on a affaire.

4 Avec tous ces personnages, le livre nous parle aussi d'une multitude d'institutions religieuses et "psy » - et tire de nombreux fils d'analyse. Comment en rendre compte? On tirera ici un seul fil susceptible d'illustrer la richesse tant des informations que des analyses : les façons $d^{\prime}$ « acculturer », $d$ '« accommoder ", les croyances catholiques et «la» psychanalyse. Je le tirerai en sociologue intéressée par le «bricolage», les «négociations » ou «transactions symboliques »-termes que l'on ne trouve pas dans le livre -, les « réinterprétations » terme, lui, bien présent.

Commençons donc par les réinterprétations. A. Demazières peut, par exemple, observer que «sont plus ou moins superposés instincts freudiens et passion thomasienne » (p.24), soulignant d'ailleurs que «le thomisme a joué un rôle majeur dans le rapprochement entre catholicisme et psychanalyse »: dans un livre écrit en 1943 Gregory Zilboorg a "rapproché » son concept d'appareil psychique de la notion aristotélico-thomiste de "sensorium commune", siège des sensations (p.78). La réinterprétation qui a sans doute eu le plus de succès concerne la substitution de la profondeur du transcendant à la profondeur des pulsions sexuelles (p. 173). Dans cette perspective, Jung et son inconscient non sexuel a reçu une attention toute particulière. En 1953, Pie XII consacre de fait une " psychologie des profondeurs » aux contours flous (p. 169). Dans cette ligne Marie Choisy, figure particulièrement active tant en France qu'à l'international, notamment en tant qu'organisatrice des Congrès catholiques internationaux de psychothérapie et de psychologie clinique, s'est faite l'avocate d'une psychologie collective spiritualiste. Le caractère poreux de la frontière entre culpabilité et péché favorise, à ses yeux, un dialogue et une complémentarité entre psychanalyse et religion: si la psychanalyse s'avère efficace pour guérir la culpabilité individuelle, elle a besoin de la religion pour soigner la culpabilité collective. Où l'on observe ici un syncrétisme - terme que je veux ici descriptif et non pas péjoratif - entre religion et psy qui aura une longue postérité.

6 Un procédé très fréquent d'articulation entre catholicisme et psychanalyse consiste à opérer des distinctions permettant de ne retenir de la psychanalyse que ce qui peut en être accepté, intégré, sans trop de dommage pour la doctrine catholique ou pour certains engagements. La première des distinctions est ainsi entre doctrine et méthode psychanalytiques. Cette distinction suppose généralement une certaine acceptation de la méthode et le refus du «pansexualisme » et de l'athéisme de la théorie freudienne. Pourtant, dans un cas fondamental il en va autrement : si la conception freudienne de la sexualité infantile peut être tolérée, ses applications pédagogiques - l'éducation sexuelle, la " pédanalyse » - sont formellement condamnées. Sont aussi distingués ce qui peut être considéré comme science - le magistère de l'Église a accordé à la nouvelle discipline un statut de science - et l'« excroissance philosophique » de la psychanalyse : 
la perception de celle-ci comme une discipline scientifique appelle à juger de sa vérité non seulement sous la lumière de la foi, mais aussi et surtout sous celle de la raison. Dès lors que la psychanalyse est ainsi insérée dans un cadre strictement scientifique, psychologique ou psychiatrique, et partant dans une éthique médicale, elle peut être moralisée dans un sens catholique (p. 239). Une autre distinction fondamentale opérée a trait à l'objet même de la psychanalyse : non pas la normalité mais le pathologique, la psychanalyse ne concerne que la pathologie. Ainsi le surmoi freudien ne concerne-t-il que les cas pathologiques, l'homme normal n'étant pas, lui, sous l'emprise du surmoi, mais gouverné par sa conscience morale: la vie spirituelle n'entre donc pas dans le champ d'examen de la psychanalyse (p. 51).

7 Le recours à la distinction tend - d'abord chez des psys, puis plus largement au fil du temps - à se faire ligne de partage, pouvant d'ailleurs permettre des complémentarités. Par exemple, Gregory Siegburg en distinguant psyché et âme permet que la religion conserve le monopole de l'âme, la psychanalyse ne s'intéressant, elle, qu'à la psyché. Françoise Dolto distingue la « conscience morale » d'ordre psychique de la « conscience religieuse vraie». La notion vraie du péché est celle qui est reçue par une conscience religieuse libérée du sentiment inconscient de culpabilité, et la structuration de la conscience morale psychologique est un préalable à la croissance spirituelle.

8 Le concordisme se repère plutôt dans l'entre-deux-guerres. Ainsi, dans la ligne de son optimisme scientifique initial, Gemelli, plaidant avec vigueur pour une autonomie des sciences et pour un engagement catholique en faveur du progrès scientifique, revendique-t-il une harmonie entre science et religion fondée sur la foi même du croyant (p. 26). Au concordisme semble par la suite plutôt se substituer ce que l'on peut appeler « affinités électives ». Par exemple lorsque Charles-Henri Nodet, se référant à la notion thomiste d'analogie, souligne les points de contact entre vie psychique inconsciente et vie morale, ce qui le conduit à souligner l'apport de la psychanalyse à la morale catholique et l'importance des premières années de la vie pour la structuration morale de l'individu (p. 90). Autre affinité élective, celle entre la pensée catholique et le holisme médical qui ouvre la médecine officielle à la dimension psychique de la maladie et donc à la psychologie et à la psychanalyse (p. 88).

Enfin, il y a des alliances stratégique ou utilitaire. C'est ainsi Gemelli qui trouve dans la psychanalyse un appui à sa campagne contre la psychologie wundtienne lorsqu'elle fait autorité dans les milieux académiques (p. 29). C'est aussi la psychanalyse qui se révèle utile quand il s'agit de juger d'enfants prétendant avoir vu des apparitions de la vierge Marie : y voir une affabulation enfantine produite par leur inconscient permet de maintenir l'origine naturelle du phénomène tout en évitant de charger les enfants.

L'ouvrage est issu de la thèse de l'auteur, et elle a manifestement dû réduire drastiquement son mémoire. Le lecteur déjà informé de l'histoire des relations entre psychanalyse et catholiques se réjouira de la masse des informations que fournit le livre, en même temps que de l'érudition, sur le catholicisme et sur les psychanalyses, dont témoigne cette jeune chercheuse. Le lecteur plus profane mais très curieux de ce dossier passionnant risque, lui, d'être submergé par toutes les informations, parfois trop brièvement commentées ou analysées et trop peu souvent reprises pour des mises en perspectives synthétiques. Le livre aurait gagné à avoir une bonne centaine de pages supplémentaires (en incluant des index), ce qui est aussi dire combien la recherche dont il rend compte est remarquable. 